\title{
Der Zusammenhang von Schülermotivation und Mathematikleistung in der Überprüfung des Erreichens der Grundkompetenzen (ÜGK) 2016
}

Boris Girnat, Universität Hildesheim

Gerda Hagenauer, Universität Salzburg

Tina Hascher, Universität Bern

Auf der Basis der Erwartungs-mal-Wert-Theorie (Eccles et al., 1983) wird anhand der Schweizer ÜGK-Daten 2016 der Zusammenhang zwischen motivationalen Variablen und Mathematikleistung bei $\mathrm{N}=11$ '131 Schülerlinnen am Ende der obligatorischen Schulzeit überprüft. Die Ergebnisse bestätigen den hohen prädiktiven Wert von Lernzielorientierungen, Selbstkonzept und Selbstwirksamkeitserwartung fïr die Mathematikleistung. Geschlechtsspezifische Unterschiede, etwa das deutlich geringere mathematische Selbstkonzept von Mädchen $\left(\mathrm{N}=5^{\prime} 423\right)$ gegenüber Jungen $\left(\mathrm{N}=5^{\prime} 708\right)$ trotz vergleichbarer Leistungen, lassen sich durch eine mehrdimensionale Erfassung der mathematischen Selbstwirksamkeitserwartung differenzieren. So lassen sich beispielsweise signifikante Unterschiede in der angewandten Mathematik, nicht jedoch in der Algebra verorten. Bezüglich der Zielorientierungen bestehen ebenfalls Geschlechterunterschiede: Jungen weisen in allen Facetten höhere Werte auf als Mädchen; allerdings sind die Effekte geringer als bei den meisten Skalen der Selbstwirksamkeitserwartung.

Der Zusammenhang von Schülermotivation und Mathematikleistung in der überprüfung des Erreichens der Grundkompetenzen (üGK) 2016

\section{Einleitung}

Die schweizerischen Bildungsstandards verwenden einen Kompetenzbegriff, der sich an Weinert (2001) orientiert: Kompetenzen sind

die bei Individuen verfügbaren oder durch sie erlernbaren kognitiven Fähigkeiten und Fertigkeiten, um bestimmte Probleme zu lösen, sowie die damit verbundenen motivationalen, volitionalen ( $\mathrm{d}$. h. absichts- und willensbezogenen) und sozialen Bereitschaften und Fähigkeiten, um die Problemlösungen in variablen Situationen erfolgreich und verantwortungsvoll nutzen zu können (Weinert, 2001, S. 27f.). 
Eine umfassende Überprüfung mathematischer (Grund-)Kompetenzen sollte sich daher nicht allein auf die mathematische Leistung beschränken, sondern auch die von Weinert genannten nicht-kognitiven Aspekte des Kompetenzbegriffs einschliessen. Ebenso wie die Bildungsstandards anderer Länder und die Bildungskonzepte der meisten internationalen Vergleichsstudien (Neubrand, 2003; OECD, 2003) unterstützen die schweizerischen Bildungsstandards diese breit gefasste Sichtweise des Kompetenzbegriffes (EDK, 2011, S. 6). Selbst wenn man den Kompetenzbegriff enger definiert, stellen nicht-kognitive Merkmale von Schüler/innen wie Interesse, Selbsteinschätzung und Motivation proximale Merkmale dar, die - im Unterschied zu distalen Faktoren wie etwa der Schultyp - relativ nahe am Kompetenzerwerb liegen.

In der vorliegenden Untersuchung werden primär zwei Ziele verfolgt: (1) Zum einen soll in einer fachspezifischen Ausdifferenzierung der Zusammenhang zwischen proximalen Schüler/innenmerkmalen und der Leistung in Mathematik anhand einer repräsentativen Stichprobe von Schweizer Schüler/innen am Ende der obligatorischen Schulzeit getestet werden. Konkret werden folgende Indikatoren der Schülerinnen- und Schülermotivation und deren Bezug zur Leistung näher betrachtet: Facetten der mathematischen Selbstwirksamkeitserwartung (SW), des mathematischen Selbstkonzepts (SK) und die Zielorientierungen. (2) Zum anderen soll untersucht werden, welche Unterschiede in den genannten Merkmalen bestehen und wie diese mit distalen Einflussfaktoren zusammenhängen. Bisher liegen für die Schweiz keine repräsentativen Daten vor, die belastbare Aussagen zu Geschlechterunterschieden, Schultypunterschieden und regionalen Unterschieden im Hinblick auf die Zielorientierungen und Subfacetten der mathematischen SW zulassen.

Eine Neuentwicklung dieser Studie ist im Besonderen die differenzierte Erfassung der mathematischen SW auf Basis von vier Dimensionen, die sich auf die typischen Inhaltskomponenten des Mathematikunterrichts der Sekundarstufe I beziehen (Geometrie, Algebra, anwendungsbezogene Mathematik und Wahrscheinlichkeitstheorie). Mit dieser Auffächerung soll eine Hypothese untersucht werden, die im Rahmen der PISA-Studie 2012 formuliert wurde:

No gender differences in confidence are observed when students are asked about doing tasks that are more abstract and clearly match classroom content, such as solving a linear or a quadratic equation. However, gender differences are striking when students are asked to report their ability to solve applied mathematical tasks. (OECD, 2013, S. 91)

Diese Hypothese konnte jedoch im Rahmen der PISA-Studie nicht zufriedenstellend untersucht werden, da die PISA-Skala zur SW eindimensional konzipiert und die eben zitierte Hypothese mit Blick auf die Auswertung einzelner Items methodologisch nicht optimal war. Demgegenüber werden in unserer Studie vier Skalen mit jeweils vier Items vorgestellt, die eine methodologisch angemessene Überprüfung dieser Hypothese sowie weiterer Hypothesen erlauben. 


\section{Theoretische Grundlagen}

Die Bedingungen, die zu lernmotiviertem Verhalten und zu Lernerfolg führen, sind vielschichtig und vernetzt. Ihre Zusammenhänge werden in der Erwartungsmal-Wert-Theorie (Eccles et al., 1983) abgebildet. Diese Theorie erklärt, wie subjektive Erfolgserwartungen und subjektive Wertzuschreibungen leistungsbezogene Wahlen und die Performanz beeinflussen. Die subjektiven Erwartungsund Wertzuschreibungen werden ihrerseits von den überdauernden Selbstschemata und Zielen der Person beeinflusst. Während angenommen werden kann, dass das mathematische SK und die mathematische SW insbesondere die Erfolgserwartungen determinieren, wirken die Zielorientierungen der Schüler/ innen vor allem auf die subjektive Wertzuschreibung. Als aggregierte Erfahrungen mit dem Lernen (Selbstschemata) haben diese Merkmale einen Einfluss auf die Leistung der Lernenden (im Überblick, siehe Rosenzweig, Wigfield \& Eccles, 2019).

Akademische SKe stellen generalisierte, fachspezifische Fähigkeitseinschätzungen dar, die Lernende aufgrund von Kompetenzerfahrungen im Unterricht entwickeln (Möller \& Köller, 2004; Möller \& Trautwein, 2015). Als kognitive Repräsentationen der eigenen Fähigkeiten können sie von Fach zu Fach unterschiedlich sein. Daher wird heute von domänenspezifischen SKen gesprochen. Das mathematikbezogene SK beruht auf dem interpersonalen Vergleich des Einzelnen mit den Fähigkeiten und Leistungen seiner/ihrer Mitschüler/innen (external frame of reference) im Fach Mathematik und auf einem intrapersonellen Vergleich mit den eigenen Leistungen in anderen Fächern (internal frame of reference; Marsh, 1986).

Basierend auf der Theorie von Bandura (2001) beschreibt die SW die subjektive Erwartung einer Person, eine spezifische Aufgabe aufgrund der eigenen Fähigkeiten erfolgreich lösen zu können. Übertragen auf das Fach Mathematik stellt sie die «Überzeugung [dar], konkrete Mathematikaufgaben erfolgreich bewältigen zu können» (Bauer et al., 2014, S. 42). Die SW kann noch spezifischer gefasst werden als lediglich mit Bezug auf ein bestimmtes Schulfach, nämlich so, dass verschiedene Subdomänen eines Faches durch unterschiedliche Skalen bzw. durch ein mehrdimensionales Modell abgedeckt werden. Es wird davon ausgegangen, dass Motivation und Leistung von einer positiven Ausprägung des mathematischen SKes und den Dimensionen der SW abhängen (Krapp \& Hascher, 2014). Sowohl vom SK als auch der SW kann man aufgrund der bisherigen Forschungslage einen wesentlichen Beitrag zur Erklärung der Mathematikleistung erwarten.

Ebenso ist anzunehmen, dass die Zielorientierungen der Schüler/innen mit der Mathematikleistung in Beziehung stehen. Zielorientierungen können als überdauernde (dispositionale) Präferenzen für bestimmte Ziele und Ergebnisse in Lern- und Leistungssituationen verstanden werden, die ihrerseits die Bewertung der jeweiligen Situation (z.B. die subjektiven Wertzuschreibungen) 
beeinflussen (Niemivirta et al., 2019, S. 570f.). Es lassen sich verschiedene Formen von Zielorientierungen unterscheiden, die von Elliot (1999) in vier Gruppen eingeteilt wurden: aufsuchende Lernzielorientierung und aufsuchende Leistungszielorientierung sowie meidende Lernzielorientierung und meidende Leistungszielorientierung. Das Konzept der «meidenden Lernzielorientierung» wurde in Arbeiten bisher eher selten aufgegriffen (Niemivirta et al., 2019). Viele Studien unterscheiden drei Zieldimensionen: Lernzielorientierung (Ziel ist es, die eigenen Fähigkeiten zu erweitern; LZO), Annäherungsleistungszielorientierung (Ziel ist es, hohe Fähigkeiten zu demonstrieren; ALZO) und Vermeidungsleistungszielorientierung (Ziel ist es, niedrige(re) Fähigkeiten zu verbergen; VLZO). Des Weiteren wird die "Arbeitsvermeidung» (AV) als Abwesenheit von lern- und leistungsmotivierten Zielen charakterisiert (Niemivirta et al., 2019; Spinath, 2009).

\section{Forschungsstand}

\section{Motivation und Mathematikleistung}

Sowohl die SW als auch das mathematische SK stellen relevante Facetten der Kompetenzüberzeugung von Schüler/innen dar. Obwohl eine inhaltliche und konzeptionelle Nähe besteht (Ahn \& Bong, 2019), lassen sie sich nicht nur theoretisch, sondern auch empirisch voneinander trennen und tragen in gemeinsamen Modellen jeweils signifikant zur Erklärung der Leistung bei (Feng et al., 2018). Der Zusammenhang zwischen der SW, dem SK und der (Mathematik-) Leistung ist üblicherweise stark (Cheema, 2018; Multon et al., 1991) und wechselseitig (Hwang et al., 2016; Marsh \& Martin, 2011). Je näher sich die Erfassung der SW oder des SKs an den Aufgaben des Leistungstests orientiert, desto enger fallen auch die Zusammenhänge aus (Kriegbaum et al., 2015).

Studien, die sich mit dem Zusammenhang zwischen den Zielorientierungen und den Leistungen von Schüler/innen befassen, zeigen heterogene Befunde. Insgesamt betrachtet sind die Zusammenhänge eher gering. Eine LZO korreliert im Allgemeinen (schwach) mit einer guten Leistung (Keys et al., 2012); in manchen Studien löst sich der Zusammenhang jedoch auf, wenn um weitere Faktoren, z. B. um die Leistungsfähigkeit zu einem früheren Zeitpunkt, kontrolliert wird (Seaton et al., 2014). Eine VLZO steht meist schwach negativ mit der Leistung in Beziehung. Die ALZO weist eher positive Beziehungen zur Leistung auf; es zeigen sich jedoch auch negative oder fehlende Zusammenhänge (im Überblick, Maehr \& Zusho, 2009; Seaton et al., 2014). Ein Blick auf diese disparaten Ergebnisse lässt vermuten, dass schwächere Zusammenhänge zwischen Zielorientierungen und Leistung zu erwarten sind als zwischen Leistung, SK und SW. 
Motivation und Mathematikleistung in Abhängigkeit von

Geschlecht, Schulform und Sprachregion

Internationale Vergleichsstudien wie z. B. PISA belegen, dass Mädchen im Vergleich zu Jungen in vielen Ländern eine geringere Mathematikleistung und eine geringere Selbsteinschätzung und Motivation zur Mathematik aufweisen. Dieser Unterschied in der Mathematikleistung zu Gunsten der Jungen ist auch in der Schweiz sichtbar und liegt signifikant über dem OECD-Schnitt (OECD, 2016). Man spricht vom «Achievement Gap» und vom «Confidence Gap». Während der "Achievement Gap» die Leistungsunterschiede zwischen Mädchen und Jungen thematisiert, wird im "Confidence Gap» auf das regelmässig geringere Vertrauen von Mädchen in die eigenen Fähigkeiten (Pajares, 1996) und die Bereitschaft, sich mit mathematischen Aufgaben zu beschäftigen, verwiesen (Ross et al., 2012).

Auch der besuchte Schultyp weist eine Bedeutung für die Motivation der Schüler/innen auf. Schüler/innen, die Schulen mit einem geringeren Anforderungsniveau besuchen, weisen (im Mittel) nicht nur eine geringere Leistungsstärke, sondern auch eine geringere Motivation auf. So konnten Buccheri, Erzinger, Hochweber und Brühwiler (2014) für die Schweiz auf Basis der PISA2012-Mathematik-Daten beispielsweise zeigen, dass in Schulen mit hohen Anforderungen nur 1\% der Schülerinnen in die Risikogruppe «leistungsschwach und sozial benachteiligt» fallen, während dies $46 \%$ in Schulen mit Grundanforderungen sind. Allerdings treten auch innerhalb eines Schultyps beträchtliche Variationen auf (siehe z.B. Köller et al., 2006).

In der Schweiz lassen sich im Hinblick auf die Mathematikleistung (PISA, 2012) Unterschiede zwischen den Sprachregionen festhalten. Carulla, Moreau und Nidegger (2014) belegten einen geringeren Anteil an Risikoschüler/innen als in den anderen beiden Sprachregionen und einen höheren Anteil an Schüler/ innen mit hoher Leistungsstärke in der Deutschschweiz, wobei diese Anteile zwischen den einzelnen Teilbereichen der Mathematikleistung variierten. Die Autor/innen geben allerdings zu bedenken, «dass sich die Zielsetzung der PISAErhebung und diejenigen der Schweizer Standards nicht decken» (S. 47). Es bleibt also zu prüfen, inwieweit sich die Leistungen der Schüler/innen in den ÜGK Testungen zwischen den Sprachregionen unterscheiden. Zudem gilt es zu untersuchen, ob sich Unterschiede auch in den Kompetenzeinschätzungen (SK und SW) und Zielorientierungen zeigen.

\section{Die vorliegende Studie}

Im Mittelpunkt der vorliegenden Studie steht die Frage, inwieweit das mathematische SK, die SW und die Zielorientierungen die mathematische Leistung der Schüler/innen erklären können. Auf Basis der Theorie und der bisherigen empirischen Befundlage werden die folgenden Hypothesen angenommen: Die Mathematikleistung ist umso höher, 
- je höher das mathematische Selbstkonzept (H1),

- je höher die mathematische Selbstwirksamkeit (in den verschiedenen Dimensionen) (H2),

- je stärker die Lernzielorientierung (H3)

- je stärker die Annäherungsleistungszielorientierung (H4),

- je schwächer die Vermeidungsleistungszielorientierung (H5) und

- je niedriger die Arbeitsvermeidung (H6) ist.

Des Weiteren wird postuliert, dass Mädchen geringere Leistungswerte (H7) sowie eine insgesamt betrachtet negativere Motivationslage für Mathematik aufweisen als Jungen (H8). Dasselbe gilt für Schüler/innen, die einen Schultyp mit niedrigeren Anforderungen besuchen im Vergleich zu Schüler/innen, die einen Schultyp mit höheren Anforderungen absolvieren (H9 für Leistung, H10 für Motivation). Aufgrund unterrichts- und schulkultureller Unterschiede werden auch für die Sprachregionen Differenzen vermutet. Es lassen sich keine Hypothesen a-priori formulieren, da Vergleichsstudien in der Schweiz bisher fehlen. Ebenso lässt sich über den Einfluss der Schulnoten auf die Leistung keine Hypothese formulieren, da sie in relevanten Studien wie PISA nicht für die Mathematikleistung, sondern nur für die Lesekompetenz erhoben werden. In diesem Fall besteht allerdings eine (moderate) Korrelation, die von Land zu Land zwischen .19 und .58 bei einem Mittelwert von .39 schwankt (OECD, 2012, S. 51).

\section{Methode}

\section{Stichprobe}

Die Revision mehrere Bildungsartikel der Bundesverfassung in Folge der Abstimmung vom 21.5.2006 hat zu einer Vereinheitlichung des schweizerischen Schulsystems und zu einer obligatorischen Überprüfung der Erreichung der Grundkompetenzen (ÜGK) in regelmässigen Abständen geführt (Konsortium ÜGK, 2019). Die erste überhaupt durchgeführte ÜGK im Jahr 2016 hat das Fach Mathematik in der 11. Jahrgangsstufe zum Gegenstand. Ihre Zielpopulation sind sämtliche Schüler/innen der 11. Jahrgangsstufe an Schweizer Schulen, die Gesamtpopulation von etwa 85'000 Personen verteilt sich auf rund 1'500 Schulen (Verner \& Helbling, 2019, S. 6). Aus der Grundgesamtheit wurde für ÜGK eine Stichprobe von 22'423 Schüler/innen in 3'552 Klassen von 830 Schulen gezogen. Dabei wurde je nach Grösse des Kantons eine Vollerhebung, eine einstufige oder eine zweistufige Ziehung (bezüglich Schulen und ggf. Klassen) durchgeführt. Die verschiedenen Ziehungsverfahren wurden durch eine Gewichtung der Schüler/innen so ausgeglichen, dass die Stichprobe als repräsentativ für die Gesamtpopulation angesehen werden kann (Verner \& Helbling, 2019, insbes. S. 11). Alle Schüler/innen bearbeiteten den Leistungstest. 
Der Kontextfragebogen lag in zwei Varianten vor. Für die vorliegende Fragestellung ist die zweite Variante relevant, die Fragen enthält, die das Fach Mathematik betreffen. Diese Variante wurde von 11'131 Schüler/innen bearbeitet und ist die Grundlage unserer Auswertung. Sie kann als ebenso repräsentativ angesehen werden wie die Gesamtstichprobe, da in jeder geprüften Klasse beide Fragebogenvarianten eingesetzt und zufällig jeweils der Hälfte der Klasse zugeteilt wurden. Innerhalb dieser Teilstichprobe verteilen sich die Schüler/innen wie folgt auf die Merkmale Geschlecht, Sprachregion und Niveaustufe: 5'423 weiblich (48.7\%), 5’708 männlich (51.3\%); 8'106 deutschsprachige Schweiz (de, 72.4\%), 2646 französischsprachige (fr, 23.9\%) und 379 italienischsprachige (it, 3.5\%); 3’387 Niveau a (grundlegendes Niveau, 30.4\%), 4’374 Niveau e (mittleres, 39.3\%) und 2'878 Niveau p (höchstes, 25.9\%).

\section{Messinstrumente}

Die eingesetzten Messinstrumente bestehen aus vier Komponenten: dem Mathematiktest, den vier Skalen zur SW, der Skala zum SK und den vier Skalen zur Zielorientierung.

Der Mathematiktest besteht aus 180 Testaufgaben, die in einem YoudenSquare-Design aus 13 Testheften mit durchschnittlich jeweils 14 Aufgaben ausgeliefert wurden. Jede Schülerin bzw. jeder Schüler bearbeitete zwei Testhefte (Angelone \& Keller, 2019). Inhaltliches Ziel des Testdesigns ist es, die Bildungsstandards für die Sekundarstufe I im Fach Mathematik repräsentativ abzuprüfen. Die Aufgaben decken alle fünf Kompetenzbereiche der Bildungsstandards für die Sekundarstufe I ab: «Daten und Zufall», "Grössen und Masse», «Funktionale Zusammenhänge», "Zahl und Variable» und «Form und Raum» - sowie fünf der acht Kompetenzaspekte: «Argumentieren und Begründen», «Darstellen und Kommunizieren», "Wissen, Erkennen und Beschreiben», «Mathematisieren und Modellieren» und "Operieren und Berechnen» (Girnat \& LinneweberLammerskitten, 2019). Zusätzlich zum Leistungstest wurden die Noten durch Selbstauskunft der Schüler/innen erhoben. Eine Anpassung an die Niveaustufen wurde nicht vorgenommen.

Ausgangspunkt für die ÜGK war zunächst die eindimensional konzipierte Skala zur mathematischen SW, die 2003 und 2012 in den PISA-Tests eingesetzt wurde (OECD, 2005, S. 291f.; OECD, 2015, S. 322). Eine explorative Faktorenanalyse dieser Skala in einer Schweizer Vorstudie hat jedoch gezeigt, dass die Eindimensionalität nicht aufrechterhalten werden kann, sondern dass diese Skala gemäss den Inhaltsbereichen des Mathematikunterrichts (Geometrie, Algebra, anwendungsbezogene Aufgaben und Wahrscheinlichkeitstheorie) in mehrere Subskalen zerfällt (Girnat, 2018). Zu ähnlichen Ergebnissen kommt eine Untersuchung der PISA-Daten von 2012 für Belgien (Oberski, 2014). Daher wird in der ÜGK 2016 die PISA-Skala durch vier Skalen ersetzt, die den mathematischen Inhaltsbereichen zugeordnet sind (siehe Tab. 1).

Zur Messung des SKs wird die Skala verwendet, die seit 2000 in den PISA- 
Tests eingesetzt wird (OECD, 2002, S. 239). Zusammen mit den drei Items zum mathematischen SK ergeben sich 19 Items, die eine leistungsbezogene Selbsteinschätzung «im engeren Sinne» operationalisieren (siehe Tab. 1). Die Items zur SW wurden mit der Frage "Wie sicher bist du, dass du die folgenden Mathematik-Aufgaben lösen könntest?» eingeleitet; im Falle des SKes wurde nach dem Grad der Zustimmung zur Aussage gefragt.

Marsh et al. (2018) machen darauf aufmerksam, dass die Unterscheidung bzw. Operationalisierung von SK- und SW-Konstrukten oftmals unter Unschärfe leidet. Eine wesentliche Quelle dieser Unschärfe ist die Möglichkeit, die Konstrukte bzw. die zugehörigen Items auf unterschiedlicher Allgemeinheitsstufe zu formulieren. Wir folgen ihrem Vorschlag, allgemein formulierte Items mit Referenzrahmen dem SK und konkrete, möglichst an typische Aufgaben gebundene Items ohne Referenzrahmen der SW zuzuordnen:

$[T]$ hese constructs were classified as either math-self-concept-like constructs or self-efficacy-like constructs, according to whether the items had a specific description of a referent against which to judge competence [...]. The three math self-concept-like constructs were: self-concept (6 items; e.g., «In math, I am a talented student"); outcome-expectancy (6 items; e.g., «I am sure to get good marks in math exams when I try hard"); generalized self-efficacy (4 items; e.g., «I am convinced that I can perform well on math tasks and in math homework"). The two math self-efficacy-like constructs were: testrelated self-efficacy (three items consisting of test items administered prior to the test in which students were asked "How confident are you that you can solve this math problem?") and functional self-efficacy ([...] e.g., "How confident are you to be able to work out the price of a T-shirt when getting 20\% off")» (Marsh et al., 2018,S. 340), wobei die vier neu entwickelten SW-Skalen dem Prinzip der "functional self-efficacy» folgen.

Wie Marsh et al. (2018) gezeigt haben, lassen sich derart definierte SK- und SW-Konzepte gut empirisch trennen, d. h. man kann davon ausgehen, dass sie tatsächlich verschiedene Konstrukte messen.

\begin{tabular}{|c|c|c|}
\hline Konstrukt (Kürzel) & Itemzahl & Beispielitem \\
\hline $\begin{array}{l}\text { mathematisches Selbstkonzept } \\
\text { (matcon) }\end{array}$ & 3 & Mathematik ist eines meiner besten Fächer. \\
\hline $\begin{array}{l}\text { *SW Algebra } \\
\text { (sw.alg) }\end{array}$ & 4 & Eine Gleichung wie $3 x+5=17$ lösen. \\
\hline $\begin{array}{l}\text { *SW Anwendungen } \\
\text { (sw.anw) }\end{array}$ & 4 & $\begin{array}{l}\text { Ausrechnen, wie viel billiger ein Fernseher bei } 30 \% \\
\text { Rabatt wäre. }\end{array}$ \\
\hline $\begin{array}{l}\text { *SW Geometrie } \\
(\text { sw.geo) }\end{array}$ & 4 & Den Flächeninhalt eines Parallelogramms berechnen. \\
\hline $\begin{array}{l}\text { *SW Wahrscheinlichkeit } \\
\text { (sw.wk) }\end{array}$ & 4 & $\begin{array}{l}\text { Die Wahrscheinlichkeit berechnen, mit einem Würfel } \\
\text { zweimal nacheinander eine } 6 \text { zu werfen. }\end{array}$ \\
\hline
\end{tabular}

*Instruktion: «Wie sicher bist du, dass du die folgenden Mathematik-Aufgaben lösen könntest? » 
Zur Messung der Zielorientierungen kam das SELLMO-Inventar (Spinath et al., 2012) zur Anwendung, das an den Mathematikunterricht angepasst wurde, indem die Wendung «In der Schule» durch die Formulierung «Im MathematikUnterricht» ersetzt und die Skalen leicht gekürzt wurden (siehe Tab. 2).

\begin{tabular}{lcl} 
Tab. 2: Skalen zu den vier Dimensionen der Zielorientierung \\
\hline Konstrukt (Kürzel) & Itemzahl & Beispielitem \\
\hline $\begin{array}{l}\text { Annäherungsleistungsziele } \\
\text { (approxgoals) }\end{array}$ & 4 & $\begin{array}{l}\text { Im Mathematik-Unterricht geht es mir darum, bessere } \\
\text { Noten zu bekommen als andere. }\end{array}$ \\
$\begin{array}{l}\text { aufsuchende Lernziele } \\
\text { (learntarget) }\end{array}$ & 4 & $\begin{array}{l}\text { Im Mathematik-Unterricht geht es mir darum, etwas } \\
\text { Interessantes zu lernen. }\end{array}$ \\
$\begin{array}{l}\text { Arbeitsvermeidung } \\
\text { (avoidwork) }\end{array}$ & 4 & $\begin{array}{l}\text { Im Mathematik-Unterricht geht es mir darum, keine } \\
\text { schwierigen Fragen oder Aufgaben lösen zu müssen. }\end{array}$ \\
$\begin{array}{l}\text { Vermeidungsleistungsziele } \\
\text { (avoidblame) }\end{array}$ & 4 & $\begin{array}{l}\text { Im Mathematik-Unterricht geht es mir darum, dass } \\
\text { niemand merkt, wenn ich etwas nicht verstehe. }\end{array}$ \\
\hline
\end{tabular}

\section{Datenaufbereitung}

Für die Analysen wurde der ÜGK Datensatz verwendet (Nidegger, 2019). Da die Auswertung der EDK die Skalen des Mathematikfragebogens unberücksichtigt lässt, mussten die Daten für diesen Beitrag neu aufbereitet werden. Zur besseren Vergleichbarkeit mit der Gesamtstichprobe wurde die Aufbereitung analog zur EDK-Auswertung vorgenommen: Der Mathematiktest wurde mit einem eindimensionalen Raschmodell skaliert (von Davier, 2016). Die Modellgütekriterien (z. B. EAP-Reliabilität 0.935, SRMR 0.0459 und MADaQ3 0.0356) liegen in einem sehr guten Bereich (Beaujean, 2014, S. 92-113). Dazu wurde das Paket «TAM» (Robitzsch et al., 2018) unter «R» (R Core Team, 2018) benutzt. Der Test prüft die mathematische Kompetenz über das gesamte Fähigkeitsspektrum reliabel $a b$ und ist nicht (wie man bei einer ÜGK vermuten könnte) auf das untere Leistungsspektrum beschränkt. Die Kontextvariablen des Fragebogens wurden in die Ziehung von 50 Plausible Values (Mislevy, 1991) aufgenommen und fehlende Fragebogendaten wurden simultan mit der Ziehung der Plausible Values imputiert (Robitzsch et al., 2016). Dabei wurde das Verfahren des Predictivee-Mean-Matching verwendet (van Buuren, 2012). Nach der Aufbereitung der Daten und Ziehung der PVs wurden sämtliche Analysen mit Strukturgleichungsmodellen durchgeführt (Loehlin \& Beaujean, 2017, S. 95-125), indem die Ergebnisse über die 50 imputierten Datensätze mit jeweils einer PV gemittelt wurden (Bruneforth et al., 2016) und die Stichprobengewichtung und das Replikationsdesign (George et al., 2016) der ÜGK mit 120 Replikationszonen von der EDK übernommen wurde. Diese Analysen wurden mit den R-Paketen «lavaan» (Rosseel, 2012) und «BIFIEsurvey» (BIFIE, 2019) durchgeführt. 


\section{Ergebnisse}

Die Ergebnisse werden in vier Abschnitten dargestellt: Zunächst wird die Reliabilität bzw. die Güte der Messmodelle der in den Tabellen 1 und 2 aufgeführten Skalen angegeben; anschliessend werden die Korrelationen der Skalen untereinander und Gruppenunterschiede bezüglich relevanter Kovariaten berichtet (Geschlecht, Schultyp und Sprachregion); schliesslich wird mit schrittweise aufgebauten linearen Regressionsmodellen untersucht, in welchem Masse die Merkmale zur Erklärung der Mathematikleistung beitragen.

\section{Model lgütekriterien}

Für jede Skala wird Cronbachs $\alpha$ angegeben. Als Modellgütekriterien werden der CFI, der TLI, der RMSEA und das SRMR angegeben. Die Interpretation dieser Werte schwankt in der Literatur. Man kann jedoch einen SRMR und RMSEA unter .08 als Kennzeichen für eine gute und unter .05 als eine sehr gute Modellpassung ansehen; der CFI und der TLI sollte nahe bei 1 liegen; zuweilen werden .95 oder .90 als untere Grenzen angeben (Beaujean, 2014, S. 92-113; Loehlin \& Beaujean, 2017, S. 70-72 und 289-298). Da die Skala "matcon» nur aus drei Items besteht, lässt sich das zugehörige Messmodell nicht isoliert testen. Daher fehlen in diesem Fall die Modellgütekriterien. Insgesamt sprechen alle Gütekriterien für den Einsatz der Skalen.

\section{Tab. 3: Reliabilität/Modellgütekriterien der Skalen}

\begin{tabular}{lccccc}
\hline Skala & Cronbachs $\alpha$ & CFI & TLI & SRMR & RMSEA \\
\hline matcon & .89 & n. v. & n. v. & n. v. & n. v. \\
sw.alg & .90 & .951 & .950 & .036 & .076 \\
sw.anw & .79 & .993 & .992 & .017 & .047 \\
sw.geo & .76 & .994 & .993 & .013 & .039 \\
sw.wk & .88 & .982 & .980 & .026 & .079 \\
approxgoals & .80 & .967 & .965 & .038 & .075 \\
learntarget & .81 & .977 & .975 & .027 & .076 \\
avoidwork & .71 & .965 & .963 & .030 & .065 \\
avoidblame & .83 & .976 & .975 & .027 & .077 \\
\hline
\end{tabular}

\section{Korrelationen}

Die Tabelle 4 enthält sämtliche latente Korrelationen, die durch ein gemeinsames Strukturgleichungsmodell geschätzt wurden (Modellfit: SRMR .052, RMSEA .042, CFI .949, TLI .942).

Die Korrelationen zwischen den «im engeren Sinne» leistungsbezogenen Variablen, d. h. dem SK und den vier SW-Skalen, sind erwartungsgemäss substantiell, allerdings nicht so hoch, dass sich die Konzepte empirisch nicht voneinander unterscheiden liessen (vgl. auch Cheema, 2018; Multon et al., 1991). 
Eine Ausnahme ist lediglich die Korrelation der SW-Skalen zur Geometrie und angewandten Mathematik, die mit .87 sehr hoch ausfällt. Ebenso zeigen die vier Skalen der Zielorientierungen relativ hohe Interkorrelationen.

Tab. 4: Korrelationen zwischen latent modellierten Selbstberichtsskalen, Mathematiktest und -note

\begin{tabular}{lcccccccccc}
\hline & Note & matcon & sw.alg & sw.anw & sw.geo & $\begin{array}{c}\text { sw.wk } \\
\text { approx- } \\
\text { goals }\end{array}$ & $\begin{array}{c}\text { learn- } \\
\text { target }\end{array}$ & $\begin{array}{c}\text { avoid- } \\
\text { work }\end{array}$ & $\begin{array}{c}\text { avoid- } \\
\text { blame }\end{array}$ \\
\hline Test & $.30^{* * *}$ & $.36^{* * *}$ & $.53^{* * *}$ & $.55^{* * *}$ & $.55^{* * *}$ & $.30^{* * *}$ & $-.10^{* * *}$ & $.20^{* * *}$ & $-.06^{* * *}$ & $-.22^{* * *}$ \\
Note & & $0.68^{* * *}$ & $.30^{* * *}$ & $.40^{* * *}$ & $.32^{* * *}$ & $.26^{* * *}$ & $.14^{* * *}$ & $.30^{* * *}$ & .01 & $-.06^{* * *}$ \\
matcon & & & $.38^{* * *}$ & $.56^{* * *}$ & $.45^{* * *}$ & $.41^{* * *}$ & $.25^{* * *}$ & $.44^{* * *}$ & $.06^{* * *}$ & $-.03^{* *}$ \\
sw.alg & & & & $.66^{* * *}$ & $.73^{* * *}$ & $.39^{* * *}$ & $.04^{* *}$ & $.37^{* * *}$ & $.04^{* *}$ & $-.12^{* * *}$ \\
sw.anw & & & & & $.87^{* * *}$ & $.69^{* * *}$ & $.16^{* * *}$ & $.49^{* * *}$ & $.11^{* * *}$ & $-.07^{* * *}$ \\
sw.geo & & & & & & $.49^{* * *}$ & $.11^{* * *}$ & $.47^{* * *}$ & $.11^{* * *}$ & $-.08^{* * *}$ \\
sw.wk & & & & & & & $.21^{* * *}$ & $.34^{* * *}$ & $.14^{* * *}$ & $.04^{*}$ \\
approxgoals & & & & & & & $.56^{* * *}$ & $.58^{* * *}$ & $.80^{* * *}$ \\
learntarget & & & & & & & & $.32^{* * *}$ & $.28^{* * *}$ \\
avoid-work & & & & & & & & & $.59^{* * *}$ \\
\hline
\end{tabular}

Interessant sind die Korrelationen zwischen diesen beiden Skalengruppen: Die LZO fügt sich mit ähnlich hohen Korrelationen gut in die leistungsbezogenen Variablen ein. Die beiden vermeidungsbezogenen Skalen haben niedrige, zum Teil negative Korrelationen. Interessant ist vor allem die Ambiguität der ALZO: Diese Skala korreliert positiv mit der Note, dem SK und den Facetten der SW, jedoch negativ mit dem Mathematiktest.

\section{Gruppenunterschiede}

Gruppenunterschiede aller Skalen wurden bezüglich des Geschlechts, des Schulniveaus und der Sprachregion geprüft (siehe Tab. 5). Das Schulniveau wird in drei Stufen unterschieden, nämlich nach grundlegenden (a), mittleren (e) und höchsten (p) Anforderungen. Im Datensatz der EDK sind ausserdem Schüler/ innen mit speziellem Förderungsbedarf als Niveau $s$ ausgewiesen $(N=492)$. Je nach Kanton werden diese Niveaustufen durch verschiedene Schulformen oder durch integrierte oder kooperative Gesamtschulen realisiert (EDK, 2017). Alle Gruppenunterschiede wurden in Cohens $d$ angegeben (Cohen, 1988). Üblicherweise interpretiert man einen Wert von 0.2 als kleinen Effekt, 0.5 als mittleren Effekt und 0.8 als grossen Effekt (Cohen, 1988). Im Fall des Geschlechts ist die Bezugsgruppe «männlich» (m) und bei der Sprachregion ist es die Deutschschweiz (de). Bei den Schulniveaus wurde in einem zweistufigen Verfahren zuerst der Unterschied zwischen dem a- und dem e-Niveau angegeben, dann derjenige zwischen dem e- und p-Niveau. Der Unterschied zwischen dem a- und p-Niveau lässt sich durch Addition der beiden Differenzen errechnen (wie im Fall der Leistungstests beispielhaft als p/a: $d=1.98^{* * *}$ angegeben). 
Bei allen Gruppenvergleichen wurde die Messinvarianz überprüft. Sie ist bezüglich des Geschlechtes und der Sprachregionen bei allen Skalen erfüllt, bei den Schulniveaus jedoch nicht mit Blick auf die Niveaustufe s. Daher wurde diese Gruppe aus allen Analysen ausgeschlossen.

\section{Tab. 5: Gruppenunterschiede in Cohens d}

\begin{tabular}{|c|c|c|c|}
\hline Skala & Geschlecht & Niveau & Sprachregion \\
\hline Modellgüte & $\begin{array}{l}\text { SRMR .055 } \\
\text { RMSEA .047 } \\
\text { CFI .975 } \\
\text { TLI .973 }\end{array}$ & $\begin{array}{l}\text { SRMR .053 } \\
\text { RMSEA .045 } \\
\text { CFI .969 } \\
\text { TLI .968 }\end{array}$ & $\begin{array}{l}\text { SRMR .055 } \\
\text { RMSEA .046 } \\
\text { CFI .975 } \\
\text { TLI .974 }\end{array}$ \\
\hline Personenzahl & $\begin{array}{l}\text { w: 5’423 } \\
\text { m: 5’708 }\end{array}$ & $\begin{array}{l}\text { a: 3’387 } \\
\text { e: 4’374 } \\
\text { p: 2'878 }\end{array}$ & $\begin{array}{l}\text { de: } 8^{\prime} 106 \\
\text { fr: } 2^{\prime} 646 \\
\text { it: } 379\end{array}$ \\
\hline Test & $\mathrm{w} / \mathrm{m}: d=-0.14^{* * *}$ & $\begin{array}{l}\mathrm{e} / \mathrm{a}: d=1.02^{* * *} \\
\mathrm{p} / \mathrm{e}: d=0.96^{* * *} \\
\mathrm{p} / \mathrm{a}: d=1.98^{* * *}\end{array}$ & $\begin{array}{l}\text { fr/de: } d=0.20^{* * *} \\
\text { it/de: } d=0.13^{* * *}\end{array}$ \\
\hline Note & $\mathrm{w} / \mathrm{m}: d=-0.20^{* * *}$ & $\begin{array}{l}\mathrm{e} / \mathrm{a}: d=0.09^{*} \\
\mathrm{p} / \mathrm{e}: d=0.00\end{array}$ & $\begin{array}{l}\mathrm{fr} / \mathrm{de}: d=-0.17^{* * *} \\
\text { it } / \mathrm{de}: d=-0.25^{* * *}\end{array}$ \\
\hline matcon & $\mathrm{w} / \mathrm{m}: d=-0.63^{* * *}$ & $\begin{array}{l}\mathrm{e} / \mathrm{a}: d=0.06^{* * *} \\
\text { p/e: } d=0.00\end{array}$ & $\begin{array}{l}\mathrm{fr} / \mathrm{de}: d=0.00 \\
\text { it } / \mathrm{de}: d=-0.23^{* * *}\end{array}$ \\
\hline sw.alg & $\mathrm{w} / \mathrm{m}: d=0.00$ & $\begin{array}{l}\text { e/a: } d=0.56^{* * *} \\
\text { p/e: } d=0.57^{* * *}\end{array}$ & $\begin{array}{l}\text { fr/de: } d=0.09^{*} \\
\text { it/de: } d=0.36^{* * *}\end{array}$ \\
\hline sw.anw & $\mathrm{w} / \mathrm{m}: d=-0.60^{* * *}$ & $\begin{array}{l}\text { e/a: } d=0.39^{* * *} \\
\text { p/e: } d=0.40^{* * *}\end{array}$ & $\begin{array}{l}\mathrm{fr} / \mathrm{de}: d=0.08 \\
\text { it/de: } d=0.25^{* * *}\end{array}$ \\
\hline sw.geo & $\mathrm{w} / \mathrm{m}: d=-0.23^{* * *}$ & $\begin{array}{l}\text { e/a: } d=0.43^{* * *} \\
\text { p/e: } d=0.57^{* * *}\end{array}$ & $\begin{array}{l}\mathrm{fr} / \mathrm{de}: d=0.52^{* * *} \\
\text { it/de: } \mathrm{d}=0.38^{* * *}\end{array}$ \\
\hline sw.wk & $\mathrm{w} / \mathrm{m}: d=-0.54^{* * *}$ & $\begin{array}{l}\mathrm{e} / \mathrm{a}: d=0.14^{* * *} \\
\text { p/e: } d=0.06\end{array}$ & $\begin{array}{l}\mathrm{fr} / \mathrm{de}: d=0.09^{*} \\
\mathrm{it} / \mathrm{de}: d=0.13^{*}\end{array}$ \\
\hline approxgoals & $\mathrm{w} / \mathrm{m}: d=-0.42^{* * *}$ & $\begin{array}{l}\text { e/a: } d=-0.22^{* * *} \\
\text { p/e: } d=-0.24^{* * *}\end{array}$ & $\begin{array}{l}\text { fr/de: } d=0.02 \\
\text { it/de: } d=0.13^{*}\end{array}$ \\
\hline learntarget & $\mathrm{w} / \mathrm{m}: d=-0.23^{* * *}$ & $\begin{array}{l}\text { e/a: } d=0.01 \\
\text { p/e: } d=0.11^{* *}\end{array}$ & $\begin{array}{l}\mathrm{fr} / \mathrm{de}: d=0.15^{* * *} \\
\text { it/de: } d=0.47^{* * *}\end{array}$ \\
\hline avoidwork & $\mathrm{w} / \mathrm{m}: d=-0.23^{* * *}$ & $\begin{array}{l}\text { e/a: } d=0.12^{* * *} \\
\text { p/e: } d=0.00\end{array}$ & $\begin{array}{l}\mathrm{fr} / \mathrm{de}: d=0.23^{* * *} \\
\text { it/de: } d=0.12^{*}\end{array}$ \\
\hline avoidblame & $\mathrm{w} / \mathrm{m}: d=-0.17^{* * *}$ & $\begin{array}{l}\text { e/a: } d=0.27^{* * *} \\
\text { p/e: } d=0.19^{* * *}\end{array}$ & $\begin{array}{l}\text { fr/de: } d=0.09^{*} \\
\text { it/de: } d=0.30^{* * *}\end{array}$ \\
\hline
\end{tabular}

Legende: $\mathrm{w}=$ weiblich; $\mathrm{m}=$ männlich (Referenzkategorie); $\mathrm{a}=$ grundlegendes Niveau (Referenzkategorie ggü. e); e = mittleres Niveau (Referenzkategorie ggü. $\mathrm{p}$ ); $\mathrm{p}=$ höchstes Niveau; de $=$ deutschsprachig (Referenzkategorie); $\mathrm{fr}$ = französischsprachig; it = italienischsprachig

Der Geschlechterunterschied liegt im Leistungstest $(d=-0.14)$ in derselben, eher «kleinen» Grössenordnung wie bei den PISA-Studien 2012 und 2015 (OECD, 2016, S. 214) und fällt zu Gunsten der Jungen aus. Die Geschlechterunterschiede im mathematischen SK sind jedoch mehr als viermal so gross $(d=-0.63)$ wie der tatsächlich festgestellte Leistungsunterschied und zeigen durchwegs niedrigere Werte bei den Mädchen. Die Unterschiede in der SW bezüglich anwendungs- 
orientierter Aufgaben ist ähnlich gross $(d=-0.60)$, Geometrie und Wahrscheinlichkeitstheorie liegen im Mittelfeld. Auch hier weisen Mädchen jeweils tiefere Werte auf. Lediglich bezüglich der Algebra ist kein Unterschied festzustellen.

Zwischen den Niveaustufen unterscheidet sich die Mathematikleistung stark $(d=1.02$ bzw. 0.96). Diese Unterschiede spiegeln sich jedoch weder in den Schulnoten noch im mathematischen SK wider. In beiden Fällen dürfte dies ein Hinweis darauf sein, dass Noten und SK nur relativ zum Umfeld der Schüler/ innen eine Aussagekraft haben, d. h. Noten niveauspezifisch vergeben werden und das SK im Vergleich zu Mitschüler/innen aufgebaut wird. Die SW - insbesondere bezüglich der Algebra - spiegelt hingegen Unterschiede niveauübergreifend wider, wenn auch nicht in derselben Grössenordnung wie die tatsächlichen Leistungsunterschiede. Auffällig unter den Skalen zur Zielorientierung ist das gegenläufige Verhalten der ALZO und der VLZO: Die Werte der ALZO fallen mit zunehmendem Niveau ab, diejenigen der VLZO steigen.

Beim Vergleich der Sprachregionen fällt auf, dass die Schüler/innen aus der französisch- und italienischsprachigen Schweiz im Leistungstest signifikant, wenn auch mit kleiner Effektstärke, bessere Ergebnisse erzielen als die Schüler/ innen aus der Deutschschweiz.

\section{Regressionsmodelle}

Als letzter Schritt der Auswertung werden latente lineare Regressionsmodelle vorgestellt, zunächst die Regressionsmodelle, die das SK und die Dimensionen der SW als Prädiktoren enthalten (Tabelle 6). Zuoberst wird zunächst das Modell mit allen Prädiktoren aufgeführt, anschließend werden nicht-signifikante Prädiktoren entfernt (zum Ausschluss der Skala sw.geo trug ausserdem die bedenklich hohe Korrelation mit der Skala sw.anw bei). Auffällig ist der unterschiedliche Beitrag der verschiedenen Subskalen der SW: Die SW zu anwendungsorientierten Aufgaben ist der stärkste Prädiktor (bis zu $\beta=.44$ ), gefolgt von der Algebra (bis zu $\beta=.28$ ) und - sehr überraschend - mit negativem Vorzeichen von der Wahrscheinlichkeitstheorie (um $\beta=-.11$ ). Bemerkenswert ist, dass die Noten und das mathematische SK im Rahmen der Gesamtstichprobe kaum zur Erklärung der Mathematikleistung beitragen, differenziert nach Schulniveaus jedoch deutlich stärker.

Im zweiten Modell werden die Zielorientierungen als Prädiktoren betrachtet (siehe Tab. 7). Die gesamte aufgeklärte Varianz ist eher niedrig (zwischen $\mathrm{R}^{2}=$ .132 und $\mathrm{R}^{2}=.228$ ). Erwartungsgemäss liefert die $\mathrm{LZO}$ stets einen positiven, relativ grossen Beitrag zur Varianzaufklärung. Dahingegen steht die VLZO negativ mit der Leistung in Beziehung. Zwei Einzelergebnisse sind bemerkenswert: Die ALZO trägt nur auf einem $p<.05-$ Niveau positiv zur Varianzaufklärung bei und ist im Allgemeinen bei Schülerinnen ein negativer Indikator (bei Schülern hingegen nicht signifikant), während bei Schülern - anders als bei Schülerinnen - die AV ein (schwach) positiver Indikator sind. 
Tab. 6: Regressionsmodelle zum Selbstkonzept (matcon) und zur Selbstwirksamkeitserwartung (sw.xx) mit der Mathematikleistung als AV

\begin{tabular}{|c|c|c|c|c|c|}
\hline Prädiktoren & $b_{i}$ & $\sigma\left(b_{\mathrm{i}}\right)$ & $\beta_{\mathrm{i}}$ (standardisiert) & $\mathrm{R}^{2}$ & SRMR/RMSEA/CFI/TLI \\
\hline sw.alg & 0.46 & 0.033 & $.25^{* * *}$ & \multirow[t]{4}{*}{.362} & \multirow[t]{4}{*}{$.044 / .055 / .983 / .982$} \\
\hline sw.anw & 0.86 & 0.114 & $.39^{* * *}$ & & \\
\hline sw.geo & 0.15 & 0.092 & & & \\
\hline sw.wk & -0.18 & 0.034 & $-.11^{* * *}$ & & \\
\hline sw.alg & 0.51 & 0.030 & $.28^{* * *}$ & \multirow{3}{*}{.356} & \multirow{3}{*}{$.037 / .052 / .992 / .989$} \\
\hline sw.anw & 0.94 & 0.055 & $.44^{* * *}$ & & \\
\hline sw.wk & -0.20 & 0.030 & $-.12^{* * *}$ & & \\
\hline sw.alg & 0.51 & 0.029 & $.28^{* * *}$ & \multirow[t]{5}{*}{.361} & \multirow[t]{5}{*}{$.037 / .052 / .992 / .990$} \\
\hline sw.anw & 0.86 & 0.060 & $.40^{* * *}$ & & \\
\hline sw.wk & -0.20 & 0.030 & $-.12^{* * *}$ & & \\
\hline Note & 0.09 & 0.025 & $.05^{* *}$ & & \\
\hline matcon & 0.07 & 0.028 & $.04^{* *}$ & & \\
\hline sw.alg & & & $\mathrm{m}: .26^{* * *} ; \mathrm{w}: .28^{* * *}$ & \multirow{5}{*}{$\begin{array}{l}\mathrm{m}: .329 \\
\mathrm{w}: .400\end{array}$} & \multirow[t]{5}{*}{$.039 / .053 / .990 / .989$} \\
\hline sw.anw & & & $\mathrm{m}: .40^{* * *} ; \mathrm{w}: .40^{* * *}$ & & \\
\hline sw.wk & & & $\mathrm{m}:-.14^{* * *} ; \mathrm{w}:-.11^{* * *}$ & & \\
\hline Note & & & $\mathrm{m}: .00 ; \mathrm{w}: .11^{* * *}$ & & \\
\hline matcon & & & m: .08***; w: .02 & & \\
\hline sw.alg & & & a: .05 ; e: $.16^{* * *}$; p: -.03 & a: .328 & \multirow[t]{5}{*}{$.043 / .052 / .989 / .988$} \\
\hline sw.anw & & & a: $.36^{* * *} ;$ e: $.27^{* * *} ;$ p: $.43^{* * *}$ & e: .383 & \\
\hline sw.wk & & & a: $-.15^{* * *} ;$ e: $.01 ;$ p: -.04 & p: .332 & \\
\hline Note & & & a: $.08^{* * *}$; e: $.03 ;$ p: $.13^{* * *}$ & & \\
\hline matcon & & & a: . $21^{* * *}$; e: $.17^{* * *}$; p: $.19^{* *}$ & & \\
\hline
\end{tabular}

Legende: $\mathrm{w}=$ weiblich; $\mathrm{m}=$ männlich; $\mathrm{a}=$ grundlegendes Niveau; $\mathrm{e}=$ mittleres Niveau; $\mathrm{p}=$ höchstes Niveau; de $=$ deutschsprachig; $\mathrm{fr}=$ französischsprachig; it $=$ italienischsprachig

Tab. 7: Regressionsmodelle zur Zielorientierung mit der Mathematikleistung als $A V$

\begin{tabular}{|c|c|c|c|c|c|}
\hline Prädiktoren & $b_{i}$ & $\sigma\left(b_{i}\right)$ & $\beta_{\mathrm{i}}$ (standardisiert) & $\mathrm{R}^{2}$ & SRMR/RMSEA/CFI/TLI \\
\hline $\begin{array}{l}\text { approxgoals } \\
\text { learntarget } \\
\text { avoidwork } \\
\text { avoidblame }\end{array}$ & $\begin{array}{l}-0.12 \\
0.53 \\
-0.11 \\
-0.41 \\
\end{array}$ & $\begin{array}{l}0.063 \\
0.039 \\
0.052 \\
0.053 \\
\end{array}$ & $\begin{array}{l}-.08^{*} \\
.31^{* * *} \\
.05^{*} \\
-.28^{* * *}\end{array}$ & .132 & $.067 / .064 / .958 / .948$ \\
\hline $\begin{array}{l}\text { approxgoals } \\
\text { learntarget } \\
\text { avoidwork } \\
\text { avoidblame }\end{array}$ & & & $\begin{array}{l}\mathrm{m}:-.02 ; \mathrm{w}:-.20^{* * *} \\
\mathrm{~m}: .28^{* * *} ; \mathrm{w}: .36^{* * *} \\
\mathrm{m:} .11^{* *} ; \mathrm{w}: .02 \\
\mathrm{m:}-. .45^{* * *} ; \mathrm{w}:-.10^{*}\end{array}$ & $\begin{array}{l}\mathrm{m}: .162 \\
\mathrm{w}: .121\end{array}$ & $.067 / .064 / .953 / .947$ \\
\hline $\begin{array}{l}\text { approxgoals } \\
\text { learntarget } \\
\text { avoidwork } \\
\text { avoidblame }\end{array}$ & & & $\begin{array}{l}\text { a: } .04 ; \text { e: } .04 ; \mathrm{p}: .15^{*} \\
\text { a: } .28^{* * *} ; \text { e: } .26^{* * *} ; \mathrm{p}: .25^{* * *} \\
\text { a: } .01 ; \text { e: } .00 ; \mathrm{p}: .02 \\
\text { a: }-.29^{* * *} ; \text { e: }-.22^{* * *} ; \text { p: } \\
-.32^{* * *}\end{array}$ & $\begin{array}{l}\text { a: } .228 \\
\text { e: } .141 \\
\text { p: } .137\end{array}$ & $.066 / .064 / .956 / .953$ \\
\hline
\end{tabular}

Legende: $\mathrm{w}=$ weiblich; $\mathrm{m}=$ männlich; $\mathrm{a}=$ grundlegendes Niveau; $\mathrm{e}=$ mittleres Niveau; $\mathrm{p}=$ höchstes Niveau; de = deutschsprachig; $\mathrm{fr}=$ französischsprachig; it $=$ italienischsprachig 
Die Tabelle 8 stellt schliesslich die Ergebnisse eines Modells dar, das alle hier betrachteten Skalen als Prädiktoren enthält. Die Steigerung in der Varianzaufklärung ist gegenüber den Modellen in Tabelle 7 eher marginal, d. h. zusätzlich zu den dort verwendeten Skalen trägt die Zielorientierung nicht viel zur Varianzaufklärung bei (für sich genommen, wie man in der Tabelle 6 sieht, jedoch schon). Nach Entfernung der Skalen, die nicht signifikant zur Varianzaufklärung beitragen, bleiben nur noch sechs Prädiktoren übrig, ohne dass sich die gesamte aufgeklärte Varianz stark verringert (von $\mathrm{R}^{2}=.401$ auf $\left.\mathrm{R}^{2}=.394\right)$. Die bedeutsamsten Prädiktoren sind die Skalen der SW zur anwendungsbezogenen Mathematik und zur Algebra, gefolgt von der ALZO, die allerdings mit einem negativen Gewicht zur Varianzaufklärung beitragen. Die fehlende Signifikanz der übrigen Skalen dürfte ein Suppressionseffekt sein (Conger, 1974), d. h. die in diesem Modell insignifikanten Skalen teilen so viel Varianz mit den verbleibenden signifikanten Skalen, dass sie über diesen gemeinsamen Varianzanteil hinaus keinen eigenständigen Aufklärungsanteil besitzen (siehe z. B. auch Seaton et al., 2014). Näheren Klärungsbedarf dürfte es zur Frage geben, warum allein die ALZO mit einem negativen Gewicht übrigbleibt.

Tab. 8: Regressionsmodelle zu allen Skalen mit der Mathematikleistung als AV

\begin{tabular}{|c|c|c|c|c|c|}
\hline Prädiktoren & $b_{i}$ & $\sigma\left(b_{\mathrm{i}}\right)$ & $\beta_{\mathrm{i}}$ (standardisiert) & $\mathrm{R}^{2}$ & SRMR/RMSEA/CFI/TLI \\
\hline $\begin{array}{l}\text { Note } \\
\text { matcon } \\
\text { sw.alg } \\
\text { sw.anw } \\
\text { sw.geo } \\
\text { sw.wk } \\
\text { approxgoals } \\
\text { learntarget } \\
\text { avoidwork } \\
\text { avoidblame }\end{array}$ & $\begin{array}{l}0.09 \\
0.12 \\
0.40 \\
0.75 \\
0.19 \\
-0.14 \\
-0.15 \\
-0.02 \\
0.00 \\
-0.03 \\
\end{array}$ & $\begin{array}{l}0.027 \\
0.030 \\
0.038 \\
0.121 \\
0.098 \\
0.033 \\
0.037 \\
0.023 \\
0.018 \\
0.033 \\
\end{array}$ & $\begin{array}{l}.06^{* *} \\
.08^{* * *} \\
.22^{* * *} \\
.34^{* * *} \\
.10 \\
-.09^{* * *} \\
.15^{* * *} \\
-.03 \\
.00 \\
.03\end{array}$ & .401 & $.052 / .042 / .969 / .964$ \\
\hline $\begin{array}{l}\text { Note } \\
\text { matcon } \\
\text { sw.alg } \\
\text { sw.anw } \\
\text { sw.wk } \\
\text { approxgoals } \\
\end{array}$ & $\begin{array}{l}0.08 \\
0.15 \\
0.48 \\
0.86 \\
-0.15 \\
-0.28 \\
\end{array}$ & $\begin{array}{l}0.047 \\
0.028 \\
0.029 \\
0.059 \\
0.029 \\
0.18\end{array}$ & $\begin{array}{l}.05^{* * *} \\
.09^{* * *} \\
.26^{* * *} \\
.40^{* * *} \\
-.09^{* * *} \\
-.19^{* * *}\end{array}$ & .394 & $.049 / .046 / .982 / .978$ \\
\hline $\begin{array}{l}\text { Note } \\
\text { matcon } \\
\text { sw.alg } \\
\text { sw.anw } \\
\text { sw.wk } \\
\text { approxgoals } \\
\end{array}$ & & & $\begin{array}{l}\text { m: } .00 ; \mathrm{w}: .10^{* * *} \\
\mathrm{~m}: .11^{* * *} ; \mathrm{w}: .06^{*} \\
\mathrm{~m}: .24^{* * *} ; \mathrm{w}: .27^{* * *} \\
\mathrm{~m}: .38^{* * *} ; \mathrm{w}: .40^{* * *} \\
\mathrm{~m}:-.09^{* * *} ; \mathrm{w}:-.09^{* * *} \\
\mathrm{~m}:-.20^{* * *} ; \mathrm{w}:-.16^{* * *}\end{array}$ & $\begin{array}{l}\mathrm{m}: .367 \\
\text { w: } .424\end{array}$ & $.051 / .047 / .978 / .975$ \\
\hline $\begin{array}{l}\text { Note } \\
\text { matcon } \\
\text { sw.alg } \\
\text { sw.anw } \\
\text { sw.wk } \\
\text { approxgoals } \\
\end{array}$ & & & $\begin{array}{l}\text { a: } .06^{*} ; \text { e: } .03 ; \text { p: } .13^{* * *} \\
\text { a: } .25^{* * *} \text {; : } .20^{* * *} \text {; p: } .21^{* * *} \\
\text { a: } .05 ; \text { e: } .15^{* * *} ; \text { p: }-.03 \\
\text { a: } .37^{* * *} \text { e: } .27^{* * *} \text { p: } .43^{* * *} \\
\text { a: }-.12^{* * *} \text {; : } .03 ; \text { p: }-.04 \\
\text { a: }-.18^{* * *} \text {; : }-.13^{* * *} \text {; : }-.08^{*}\end{array}$ & $\begin{array}{l}\text { a: } .328 \\
\text { e: } .299 \\
\text { p: } .258\end{array}$ & $.053 / .047 / .981 / .979$ \\
\hline
\end{tabular}




\section{Diskussion und Fazit}

Der vorliegende Beitrag hatte zum Ziel, die Rolle motivationaler Faktoren für die Mathematikleistung Schweizer Sekundarschüler/innen näher zu klären. Die theoretische Basis bildete die Erwartungs-mal-Wert-Theorie (Wigfield \& Eccles, 2000). Anhand repräsentativer Daten der ÜGK 2016 wurde getestet, wie das generelle mathematische Selbstkonzept (H1), vier fachbezogene Dimensionen der mathematischen Selbstwirksamkeit $(\mathrm{H} 2)$ und vier Zielorientierungen (H3-H6) mit der Testleistung sowie der Mathematiknote zusammenhängen. Zudem wurden Gruppenunterschiede hinsichtlich Geschlecht (H7, H8), Schultyp (H9, H10) sowie Sprachregion untersucht.

Entgegen der Erwartung (H1) trägt das mathematische SK im Rahmen der Gesamtstichprobe kaum zur Erklärung der Mathematikleistung bei, wenn auch die SW in den Modellen berücksichtigt wird. Es differenziert jedoch deutlich stärker, wenn das Schulniveau einbezogen wird. Dieser Befund legt die Annahme nahe, dass das SK eine als niveaurelative Variable zu verstehen ist, also von der Referenzgruppe beeinflusst wird (Marsh et al., 2018) und - ähnlich wie bei Schulnoten - interpersonale Vergleichsprozesse eine stärkere Rolle spielen. Es wäre deshalb interessant, in künftigen Forschungsarbeiten der Entwicklung referenzgruppensensibler Konstrukte wie generelles Fach-Selbstkonzept und Notengebung weiter nachzugehen.

Zum ersten Mal im Rahmen von Large-Scale-Assessments wurde anstelle einer eindimensionalen Skala ein mehrdimensionaler Skalenverbund zur Messung der mathematischen SW eingesetzt, der sich inhaltlich an verschiedenen Inhaltsbereichen des Mathematikunterrichts orientiert. Es zeigte sich erwartungskonform $(\mathrm{H} 2)$, dass die Skalen einen bedeutsamen prädiktiven Wert für die Ergebnisse des mathematischen Leistungstests aufweisen - insbesondere die Subskalen zur anwendungsbezogenen Mathematik und zur Algebra. Der Beitrag der verschiedenen Subskalen der SW ist allerdings unterschiedlich und die negative Bedeutung der Wahrscheinlichkeitstheorie erstaunlich. Es ist jedoch unwahrscheinlich, dass der negative Einfluss ein statistisches Artefakt z. B. aufgrund von Multikollinearität ist, da die Korrelationen zu den anderen SW-Skalen eher moderat sind. Auch spricht ein VIF-Index von 1.87 empirisch gegen das Vorliegen von Multikollinearität (Kock \& Lynn, 2012). Möglicherweise liegt eine Erklärung darin, dass Wahrscheinlichkeitstheorie traditionell kein Schwerpunkt im schweizerischen Mathematikunterricht der Sekundarstufe ist und sich gerade leistungsschwächere Schüler/innen mit Blick auf die eher einfach und alltäglich wirkenden Items auf dieser Skala überschätzen. Auf Grundlage der Konstruktion des Mathematiktests lassen sich die Unterschiede zwischen den verschiedenen SW-Skalen nicht erklären, da der Test aus all diesen Bereichen in einem ähnlichen Umfang Aufgaben enthält. Plausibel könnte die These sein, dass Schüler/innen speziell im Bereich der Algebra und der angewandten Mathematik besser zu einer Selbsteinschätzung fähig sind, die ihrer Leistungsfä- 
higkeit entspricht, als in anderen Subdomänen des Fachs, am wenigsten in der Wahrscheinlichkeitstheorie. Dies müsste jedoch erst durch weitere Forschung geprüft werden.

Wie erwartet, erlaubt ein mehrdimensionales Konzept der SWen zudem eine informativere Analyse der Geschlechterunterschiede als eine eindimensionale Skala, wie sie u. a. in den PISA-Tests eingesetzt wird (Girnat, 2018): Beispielsweise lässt sich so feststellen, dass es bezüglich der Algebra keine signifikanten Geschlechterunterschiede gibt, die Geschlechterunterschiede bezüglich der anwendungsbezogenen Mathematik am grössten sind und in diesem Fall - wie beim inhaltsbereichsübergreifenden SK - rund viermal so hoch liegen wie der durch den Mathematiktest festgestellte Leistungsunterschied. Dies unterstützt (zumindest für Schweizer Schüler/innen) die in der PISA-Studie 2012 formulierte, aber dort durch die eindimensionale Anlage der Skala nicht prüfbare These, dass in den Teilbereichen der Mathematik die Geschlechterunterschiede deutlich verschieden ausgeprägt sind (OECD, 2013, S. 91). Des Weiteren scheinen die Skalen zur SW - anders als das mathematische SK - weniger niveaurelativ zu sein, d. h. Leistungsunterschiede zwischen den Schulniveaus spiegeln sich in den Skalen zur SW wider. Der Grund dürfte darin liegen, dass die Skalen zur SW über konkrete Aufgaben abgefragt wurden, das SK dagegen auf einen sozialen Referenzrahmen, z. B. die jeweilige Schulklasse, ausgerichtet ist (Marsh, 1986). Demgegenüber scheint die Schwierigkeit der Aufgaben in den themenbezogenen SW-Skalen wie ein Ankerpunkt zu wirken, der einen niveauübergreifenden Vergleich ermöglicht (vgl. auch Krapp et al., 2014).

Die Ergebnisse belegen zudem die Bedeutung der Lernzielorientierung von Schüler/innen für die Mathematikleistung (H3), während die weiteren Zielorientierungen (H4-H6) wenig bis nichts zur inkrementellen Varianzaufklärung beitragen. Womöglich lässt sich dieser Befund darin begründen, dass Schüler/ innen unterschiedliche Ziele gleichzeitig verfolgen, die zudem an die Kontrollüberzeugungen gebunden sind. Zukünftige Analysen könnten im Sinne von stärker personenzentrierten Analysen motivationale Profile von Schüler/innen und deren Effekt auf die Schulleistungen testen (z.B. Niemivirta et al., 2019). Möglicherweise müsste aber auch die Theorie der Zielorientierungen fachspezifisch weiterentwickelt werden. Dafür spricht z.B. das Ergebnis, dass Kompetenzüberzeugungen (SW) zu Teilbereichen der Mathematik stärker mit der Mathematikleistung zusammenhängen als inhaltsbereichsübergreifende Formulierungen, so wie sie auch die Skalen zu Zielorientierungen aufweisen (siehe z.B. Lazarides \& Ittel, 2019).

Wie erwartet, zeigen Mädchen tiefere Leistungswerte (H7) und eine geringere generelle Motivationslage in Mathematik als Jungen (H8). Die motivationalen Unterschiede betreffen sowohl die Leistungsziel- als auch die Lernzielorientierung. Mädchen scheinen sowohl weniger auf Anreize durch soziale Vergleiche anzusprechen als auch weniger auf persönlichen Lerngewinn. Während bereits bekannt ist, dass das Sich-Messen im Hinblick auf die Leistung mit anderen in 
der Klasse für Mädchen eine weniger relevante Motivationslage darstellt als für Jungen (Benölken, 2014; SKBF, 2018), scheint bei Schweizer Mädchen ebenfalls weniger Interesse an der Verbesserung der eigenen Kompetenzen vorzuliegen. Dieser Befund unterstreicht die Notwendigkeit eines motivationsunterstützenden Mathematikunterrichts (z.B. Rakoczy, 2008), in dem die Motivation der Mädchen gefördert wird, und entsprechende Forschungsbegleitung zur Testung der Wirksamkeit solcher Massnahmen im Rahmen von Interventionsstudien für die Sekundarstufe (siehe z.B. Brandenberger et al., 2017; Hascher et al., 2019).

Ebenso bestätigt sich, dass Schüler/innen, die einen Schultyp mit höheren Anforderungen besuchen, eine höhere Mathematikleistung zeigen (H9), sowie auch die Dimensionen der SW stärker ausgeprägt sind. Interessanterweise gibt es im Hinblick auf das SK keine substanziellen Unterschiede. Die Unterschiede im Hinblick auf die Zielorientierungen (H10) folgen ebenso keinem völlig klaren Muster: Tendenziell zeigen Schüler/innen, die Schulen des höchsten Schultyps besuchen, die positivste Motivationslage (= höchstes Ausmass an LZO); allerdings berichten sie gleichzeitig auch von einem hohen Ausmaß an VLZO. Die hohe Ausprägung der ALZO könnte auf einen hohen Leistungsdruck an Schultypen mit hohen Anforderungen hinweisen. Das höchste Ausmaß an Arbeitsvermeidung berichten Schüler/innen, die einen Schultyp auf grundlegendem Niveau besuchen; gleichzeitig ist auch ihre ALZO geringer. Interventionen zur Förderung von Motivation im Mathematikunterricht sollten daher insbesondere an Schulen mit Grundanforderungen ansetzen (Brandenberger et al., 2017).

Erstmalig können sprachregionale Vergleiche in der Motivation der Schüler/ innen auf Basis der Zielorientierungstheorie berichtet werden. Erwartungsgemäss finden sich Unterschiede und dabei sticht vor allem die deutlich höhere Lernzielorientierung von Schüler/innen aus der italienischsprachigen Schweiz im Vergleich zur deutschsprachigen Schweiz hervor. Allerdings ist auch deren AV und die VLZO höher. Worin dieser Unterschied begründet liegt, kann mit den vorliegenden Analysen nicht geklärt werden. Zukünftige Analysen sollten einen näheren Blick auf die Charakteristika des Mathematikunterrichts in den drei Sprachregionen und deren Relevanz für die Motivationslage der Schüler/innen richten, um diesen Befund erklären zu können.

Mit Blick auf die Sprachregionen ist auch zu erwähnen, dass sich die regionalen Unterschiede in den Mathematikleistungen bei der ÜGK Testung 2016 nicht mit den PISA-Ergebnissen decken, da bei ÜGK die Schüler/innen der Deutschschweiz nicht die besseren Leistungen zeigen (Konsortium PISA.ch, 2014, S. 33-48). Wie aber schon das Konsortium PISA.ch feststellt (2014, S. 34), sind die Kompetenzraster der PISA-Studie und der Bildungsstandards zwar ähnlich, aber nicht deckungsgleich, d. h. die ÜGK prüft die Mathematikleistung anders, und zwar mit einem stärkeren Bezug zu national vereinbarten Bildungszielen als die PISA-Studien, die mit der «mathematical literacy» ein eigenes, länderunabhängiges Konzept mathematischer Bildung verfolgen (Neubrand, 2003). 
Aufgrund des Querschnittdesigns von ÜGK lassen sich keine Aussagen zu den Entwicklungen von motivationalen Variablen und Lernergebnissen auf der Sekundarstufe ableiten. Zu bedenken ist zudem, dass bei ÜGK das Monitoring von Leistungsergebnissen und nicht mögliche Erklärungsfaktoren auf Unterrichtsebene (erhoben im sog. Kontextfragebogen) im Mittelpunkt stehen. Trotz der repräsentativen Stichprobe bleiben die Erkenntnisse für die Motivationsund die Mathematikdidaktikforschung damit eingeschränkt.

\section{Literatur}

Ahn, H. S., \& Bong, M. (2019). Self-efficacy in learning. In K. A. Renninger, \& S. E. Hidi (Eds.), The Cambridge Handbook of motivation and learning (pp. 63-86). Cambridge University Press.

Angelone, D. \& Keller, F. (2019). Überprüfung des Erreichens der Grundkompetenzen (ÜGK) im Fach Mathematik im 11. Schuljahr. Technische Dokumentation zur Testentwicklung und Skalierung. Aarau: Geschäftsstelle der Aufgabendatenbank EDK (ADB). http://uegkschweiz.ch/wp-content/uploads/2019/05/\%C3\%9CGK2016_Technischer-Bericht_ ADB.pdf

Bandura, A. (2001). Social-cognitive theory. Annual Review of Psychology, 52, 1-26.

Bauer, C., Ramseier, E. \& Blum-Giger, D. (2014). PISA 2012: Porträt des Kantons Bern (deutschsprachiger Teil). Forschungsgemeinschaft PISA Deutschschweiz.

Beaujean, A. (2014). Latent variable modeling using $R-A$ step-by-step guide. Routledge.

Benölken, R. (2014). Begabung, Geschlecht und Motivation. Journal für MathematikDidaktik, 35(1), 129-158.

Brandenberger, C., Hagenauer, G., \& Hascher, T. (2017). Promoting students' self-determined motivation in maths: Results of a one-year classroom intervention. European Journal of Psychology of Education, 33(2), 295-317.

BIFIE (2019). BIFIEsurvey: Tools for survey statistics in educational assessment. R package version 3.1-33. https://CRAN.R-project.org/package=BIFIEsurvey

Bruneforth, M., Oberwimmer, K. \& Robitzsch, A. (2016). Reporting \& Analysen. In S. Breit \& C. Schreiner (Hrsg.), Large-Scale Assessment mit R: Methodologische Grundlagen der österreichischen Bildungsstandardüberprüfung (S. 333-362). facultas.

Buccheri, G., Erzinger, A. B., Hochweber, J. \& Brühwiler, C. (2014). Resilienz - hohe Leistungen trotz sozial benachteiligter Herkunft. In Konsortium PISA.ch (Hrsg.), PISA 2012. Vertiefende Analysen (S. 21-31). SBFI/EDK und Konsortium PISA.ch. https://pisa. educa.ch/sites/default/files/20140923/pisa2012_vertiefende_analysen_0.pdf.

Carulla, C., Moreau, J. \& Nidegger, C. (2014). Grundkompetenzen in Mathematik und Mathematikunterricht. In Konsortium PISA.ch (Hrsg.), PISA 2012. Vertiefende Analysen (S. 33-48). SBFI/EDK und Konsortium PISA.ch. https://pisa.educa.ch/sites/default/ files/20140923/pisa2012_vertiefende_analysen_0.pdf

Cheema, J. R. (2018). Effects of math-specific self-efficacy on math literacy: Evidence from a Greek survey. Research in Education, 102(1), 13-36.

Cohen, J. (1988), Statistical power analysis for the behavioral sciences (2nd ed.). Lawrence Erlbaum.

Conger, A. (1974). A revised definition for supressor variables: A guide to their identification and interpretation. Educational and Psychological Measurement, 24, 35-46.

Eccles, J., Adler, T. F., Futterman, R., Goff, S. B., Kaczala, C. M., Meece, J., \& Midgley, C. (1983). Expectancies, values and academic behaviors. In J. T. Spence (Ed.), Achievement and achievement motives: Psychological and sociological approaches (pp. 75-146). W. H. Freeman. 
EDK (2011). Grundkompetenzen für die Mathematik. Nationale Bildungsstandards. Freigegeben von der EDK-Plenarversammlung am 16. Juni 2011. EDK. http://edudoc.ch/ record/96784/files/grundkomp_math_d.pdf, abgerufen am 24.3.2019.

EDK (2017). Schulmodelle (Sekundarstufe I). EDK. https://edudoc.educa.ch/static/strukturdaten/pdf_visualisierung_d/10_v_Schulmodell_d.pdf

Elliot, A. J. (1999). Approach and avoidance motivation and achievement goals. Educational Psychologist, 34(3), 169-189. https://doi.org/10.1207/s15326985ep3403_3

Feng, X., Wang J.-L. \& Rost, D. H. (2018). Akademische Selbstkonzepte und akademische Selbstwirksamkeiten: Interdependenzen und Beziehungen $\mathrm{zu}$ schulischen Leistungen. Zeitschrift für Pädagogische Psychologie,32(1-2), 23-38.

Girnat, B. (2018). The PISA mathematics Self-Efficacy Scale: Questions of dimensionality and a latent class concerning Algebra. In H. Palmér, \& J. Skott J. (Eds.), Students' and teachers' values, attitudes, feelings and beliefs in Mathematics classrooms (pp. 89-99). Springer.

Girnat, B. \& Linneweber-Lammerskitten, H. (2019). Schlussbericht zur Entwicklung mathematischer Testitems für die Überprüfung der Grundkompetenzen der Jahrgangsstufe 11 in Mathematik auf der Grundlage des HarmoS Kompetenzmodells Mathematik. http://uegk-schweiz. ch/wp-content/uploads/2019/06/Schlussbericht_Itementwicklung_HarmoS_UEGK.pdf

George, A. C., Oberwimmer, K. \& Itzlinger-Bruneforth (2016). Stichprobenziehung. In S. Breit \& C. Schreiner (Hrsg.), Large-Scale Assessment mit R: Methodologische Grundlagen der österreichischen Bildungsstandardüberprüfung (S. 51-82). facultas.

Hwang, M. H., Choi, H. C., Lee, A., Culver, J. D., \& Hutchison, B. (2016). The relationship between self-efficacy and academic achievement: A 5-year panel analysis. Asia Pacific Educational Researcher, 25(1), 89-98.

Hascher, T., Fries, S. \& Hagenauer, G. (2019). Grundlagen der Förderung von Motivation. In H. Gaspard, U. Trautwein \& M. Hasselhorn (Hrsg.), Jahrbuch Tests und Trends zu Motivation und Volition im Schulkontext (S. 145-160). Waxmann.

Keys, T. D., Conley, A-M. M., Duncan, G. J., \& Domina, T. (2012). The role of goal orientations for adolescents' mathematics achievement. Contemporary Educational Psychology, 37, 47-54.

Kock, N., \& Lynn, G. S. (2012): Lateral collinearity and misleading results in variance-based SEM: An illustration and recommendations. Journal of the Association for Information Systems, 13, 7, 546-580.

Konsortium PISA.ch (2014). PISA 2012: Vertiefende Analysen. Bern, Neuchâtel: SBFI/EDK und Konsortium PISA.ch.

Konsortium ÜGK (Hrsg.). (2019). Überprüfung der Grundkompetenzen. Nationaler Bericht der ÜGK 2016: Mathematik 11. Schuljahr. EDK und SRED. https://edudoc.ch/ record $/ 204075 ? \ln =\mathrm{de}$

Köller, O., Daniels, Z., Schnabel, K., \& Baumert, J. (2000). Kurswahlen von Mädchen und Jungen im Fach Mathematik: Zur Rolle von fachspezifischem Selbstkonzept und Interesse. Zeitschrift für Pädagogische Psychologie, 14(1), 26-37.

Köller, O., Trautwein, U., Lüdtke, O. \& Baumert, J. (2006). Zum Zusammenspiel von schulischer Leistung, Selbstkonzept und Interesse in der gymnasialen Oberstufe. Zeitschrift für Pädagogische Psychologie, 20(1/2), 27-39.

Krapp, A., Geyer, C. \& Lewalter, D. (2014). Motivation und Emotion. In A. Krapp \& T. Seidel (Hrsg.), Pädagogische Psychologie (S. 193-222). Beltz.

Krapp, A. \& Hascher, T. (2014). Theorien der Lern- und Leistungsmotivation. In L. Ahnert (Hrsg.), Theorien in der Entwicklungspsychologie (S. 252-281). Springer.

Kriegbaum, K., Jansen, M., \& Spinath, B. (2015). Motivation: A predictor of PISA's mathematical competence beyond intelligence and prior achievement. Learning and Individual Differences, 43, 140-148.

Lazarides, R. \& Ittel, A. (2019). Entwicklung motivationaler Orientierungen in den 
MINT-Bereichen im mittleren Jugendalter. In B. Kracke \& P. Noack (Hrsg.), Handbuch Entwicklungs- und Erziehungspsychologie (S. 369-386). Springer.

Loehlin, J. C., \& Beaujean, A. A. (2017). Latent variable models - An introduction to factor, path, and structural equation analysis. Routledge.

Maehr, M. L., \& Zusho, A. (2009). Achievement goal theory: The past, present and future. In K. R. Wentzel, \& A. Wigfield (Eds.), Handbook of motivation at school (pp. 77-104). Routledge.

Marsh, H. W. (1986). Verbal and math self-concepts. An internal/external frame of reference model. American Educational Research Journal, 23(1), 129-149.

Marsh, H. W., \& Martin, A. J. (2011). Academic self-concept and academic achievement: Relations and causal ordering. British Journal of Educational Psychology, 81, 59-77.

Marsh, H. W., Pekrun, R., Parker, P. D., Murayama, K., Guo, J., Dicke, T., \& Arens, A. K. (2018). The murky distinction between self-concept and self-efficacy: Beware of lurking jingle jangle fallacies. Journal of Educational Psychology, 111 (2), 331353.

Mislevy, R. J. (1991). Randomization-based inference about latent variables from complex samples. Psychometrika, 56(2), 177-196.

Möller, J. \& Trautwein, U. (2015). Selbstkonzept. In E. Wild \& J. Möller (Hrsg.), Pädagogische Psychologie (2. Aufl.) (S. 177-199). Springer.

Möller, J. \& Köller, O. (2004). Die Genese akademischer Selbstkonzepte: Effekte dimensionaler und sozialer Vergleiche. Psychologische Rundschau, 55, 19-27.

Multon, K. D., Brown, S. D., \& Lent, R. W. (1991). Relations of self-efficacy beliefs to academic outcomes. Journal of Counseling Psychology, 38(1), 30-38.

Neubrand, M. (2003). "Mathematical literacy"/»Mathematische Grundbildung» - Der Weg in die Leistungstests, die mathematikdidaktische Bedeutung, die Rolle als Interpretationshintergrund für den PISA-Test. Zeitschrift für Erziehungswissenschaft, 6, 338-356.

Nidegger C. (2019). ÜGK / COFO /VECOF 2016. Competencies of Swiss pupils in mathematics (Dataset). https://doi.org/10.23662/FORS-DS-1004-1

Niemivirta, M., Pulkka, A.-T., Tapola, A., \& Tuominen, H. (2019). Achievement goal orientations. A person-oriented approach. In K. A. Renninger, \& S. E. Hidi (Eds.), The Cambridge Handbook of motivation and learning (pp. 566-616). Cambridge University Press.

Oberski, D. L. (2014). lavaan.survey: An R Package for Complex Survey Analysis of Structural Equation Models. Journal of Statistical Software, 57(1), 1-27.

OECD (2002). PISA 2000 - Technical report. OECD Publishing. https://doi. org/10.1787/19963777en.

OECD (2003).PISA2003 assessmentframework: Mathematics, reading, scienceandproblemsolving knowledge and skills. OECD Publishing. http://dx.doi.org/10.1787/9789264101739-en.

OECD (2005). PISA 2003 - Technical report. OECD Publishing. https://doi. org/10.1787/19963777

OECD (2012), Grade Expectations: How Marks and Education Policies Shape Students' Ambitions, PISA, OECD Publishing. http://dx.doi.org/10.1787/9789264187528-en

OECD (2013). PISA 2012 Results: Ready to learn: Students' engagement, drive and self-beliefs (Volume III). OECD Publishing. http://dx.doi.org/10.1787/9789264201170-en.

OECD (2015). PISA 2012 - Technical report. OECD Publishing. http://dx.doi. org/10.1787/9789264208780-en.

OECD (2016). Die Leistungen 15-Jähriger in Mathematik. In PISA 2015 Results (Volume I): Excellence and equity in education. OECD Publishing. https://doi.org/10.1787/789264267879-9-de.

Pajares, F. (1996). Self-efficacy beliefs in academic settings. Review of Educational Research, 66, 543-578.

R Core Team (2018). R: A language and environment for statistical computing. R Foundation for Statistical Computing. Vienna. https://www.R-project.org/. 
Rakoczy, K. (2008). Motivationsunterstützung im Mathematikunterricht. Waxmann.

Robitzsch, A., Pham, G. \& Yanagida, T. (2016). Fehlende Daten und Plausible Values. In S. Breit \& C. Schreiner (Hrsg.), Large-Scale Assessment mit R: Methodologische Grundlagen der österreichischen Bildungsstandardüberprüfung (S. 259-294). facultas.

Robitzsch, A., Kiefer, T., \& Wu, M. (2018). TAM: Test analysis modules. R package version 3.0-21. https://CRAN.R-project.org/package=TAM.

Rosenzweig, E. Q., Wigfield, A., \& Eccles, J. S. (2019). Expectancy-value theory and its relevance for student motivation and learning. In K. A. Renninger, \& S. E. Hidi (Eds.), The Cambridge Handbook of Motivation and Learning (pp. 617-644). Cambridge University Press.

Ross, J. A., Bruce, C. D., \& Scott, G. (2012). The gender confidence gap in fractions knowledge: Gender differences in student belief-achievement relationships. School Science and Mathematics, 112(5), 278-288.

Rosseel, Y. (2012). lavaan: An R Package for Structural Equation Modeling. Journal of Statistical Software, 48(2), 1-36. http://www.jstatsoft.org/v48/i02/.

Seaton, M., Parker, P., Marsh, H. W., Craven, R. G., \& Yeung, A. S. (2014). The reciprocal relations between self-concept, motivation and achievement: Juxtaposing academic self-concept and achievement goal orientations for mathematics success. Educational Psychology, 34(1), 49-72.

Schweizerische Koordinationsstelle für Bildungsforschung (SKBF). (2018). Bildungsbericht Schweiz 2018. Schweizerische Koordinationsstelle für Bildungsforschung (SKBF).

Spinath, B. (2009). Zielorientierungen. In V. Brandstätter \& J. H. Otto (Hrsg.), Handbuch der Allgemeinen Psychologie. Motivation und Emotion (S. 64-71). Hogrefe.

Spinath, B., Stiensmeier-Pelster, J., Schöne, C. \& Dickhäuser, O. (2012). SELLMO - Skalen zur Erfassung der Lern- und Leistungsmotivation (2. Auflage). Hogrefe.

Van Buuren, S. (2012). Flexible imputation of missing data. CRC Press.

Verner, M. \& Helbling, L. (2019). Sampling ÜGK 2016 Technischer Bericht zu Stichprobendesign, Gewichtung und Varianzschätzung bei der Überprïfung des Erreichens der Grundkompetenzen 2016. Institut für Bildungsevaluation. http://uegk-schweiz.ch/ wp-content/uploads/2019/05/\%C3\%9CGK2016_Verner_Helbling_2019_-Sampling\%C3\%9CGK-2016.pdf

Von Davier, M. (2016). Rasch Model. In W. J. Van den Linden (Ed.), Handbook of item response theory - volume one: Models (pp. 31-50). CRC Press.

Weinert, F. E. (2001). Vergleichende Leistungsmessung in Schulen - eine umstrittene Selbstverständlichkeit. In F. E. Weinert (Hrsg.), Leistungsmessung in Schulen (S. 17-31). Beltz.

Wigfield, A., \& Eccles, J. S. (2000). Expectancy-value theory of achievement motivation. Educational Psychologist, 25, 68-81.

Schlagworte: Mathematikleistung; Motivation; Selbstkonzept; Selbstwirksamkeitserwartungen; Erwartungs-mal-Wert-Theorie 


\title{
Le rapport entre la motivation des élèves et leurs performances en mathématique dans la vérification de l'atteinte des compétences fondamentales (COFO) 2016
}

\begin{abstract}
Résumé
Basé sur le modèle d'expectation-valeur (Eccles et al., 1983) la relation entre les variables motivationnelles et les résultats en mathématique chez $\mathrm{N}=11$ '131 élèves est examinée à la fin de la scolarité obligatoire au moyen des données suisses de COFO 2016. Les résultats confirment la valeur prédictive élevée de la focalisation sur l'objectif de la formation, de la conception de soi et de l'autoperception de l'efficacité personnelle pour la réussite en mathématique. Les différences de genre, telles que la conception de soi en mathématique nettement inférieure des filles $\left(\mathrm{N}=5^{\prime} 423\right)$ malgré des performances comparables à celles des garçons $\left(\mathrm{N}=5^{\prime} 708\right)$, peuvent être différenciées par un recensement multidimensionnel de l'autoperception de l'efficacité personnelle en mathématiques, par exemple en situant des différences significatives en mathématiques appliquées, mais pas en algèbre. Il existe également des différences entre les sexes en ce qui concerne la focalisation sur les objectifs de formation : les garçons obtiennent des résultats plus élevés que les filles pour tous les aspects; cependant, les effets sont plus faibles que dans la plupart des échelles d'attente d'auto-efficacité.
\end{abstract}

Mots clés: Performances en mathématique, motivation, conception de soi, autoperception de l'efficacité personnelle, modèle d'expectation-valeur

\section{II rapporto tra la motivazione degli studenti e le prestazioni matematiche nella VeCoF 2016}

\section{Riassunto}

Sulla base della teoria dell'aspettativa-valore (Eccles et al., 1983) viene esaminato mediante i dati svizzeri della $\mathrm{VeCoF} 2016$ il rapporto tra variabili motivazionali e risultati matematici di 11'131 alunni al termine della scuola dell'obbligo. I risultati confermano l'alto valore predittivo dell'orientamento agli obiettivi di apprendimento, del concetto di sé e del senso di auto-efficacia sulle prestazioni matematiche. Le differenze specifiche di genere riferibili al concetto di sé matematico delle ragazze $(\mathrm{N}=5$ '423) notevolmente più debole rispetto a quello dei ragazzi nonostante le prestazioni paragonabili $\left(N=5^{\prime} 708\right)$, possono essere argomentate attraverso analisi multidimensionali relative al senso di autoefficacia in matematica, individuando ad esempio differenze significative nel dominio della matematica applicata, ma non in quello dell'algebra. Si rilevano inoltre differenze tra ragazzi e ragazze nell'orientamento degli obiettivi: i primi mostrano valori più elevati rispetto alle seconde in tutti i campi. Tuttavia, gli effetti sono inferiori rispetto a quanto rilevato nella maggior parte delle scale di autoefficacia. 
Parole chiave: Prestazioni matematiche; motivazione; concetto di sé; autoefficacia; teoria dell'aspettativa-valore

\section{The relationship between student motivation and mathematics achievement in the Assessment of Basic Competencies (ÜGK) 2016}

\section{Summary}

Based on the expectancy-value-theory (Eccles et al., 1983), we tested the relationship between motivation variables and mathematics achievement using ÜGK 2016 data of $N=11$ '131 students at the end of compulsory education. The results confirm the predictive power of learning goal-orientation, self-concept, and self-efficacy beliefs for explaining mathematics achievement. Genderspecific differences such as the pronounced lower mathematics self-concept of girls despite comparable achievement can be explained by a multi-dimensional assessment of mathematics self-efficacy beliefs as there are significant differences in applied mathematics but not in algebra.

Keywords: Mathematic achievement; motivation; self-concept; self-efficacy beliefs; expectancy-value-theory

Boris Girnat, Prof. Dr. rer. nat., Professor für Mathematik an der Universität Hildesheim, Leiter der Abteilung Mathematikdidaktik 2. Mitwirkung bei der Erstellung der Testitems und der Entwicklung des Kontextfragebogens der Überprüfung des Erreichens der Grundkompetenzen (ÜGK). Forschungsschwerpunkte: Modellierung mathematischer Kompetenzen, Einfluss von Überzeugungen, Einstellungen, Affekten und Emotionen auf das Lernen von Mathematik, digitale Medien im Mathematikunterricht und Schulbuchentwicklung.

Universität Hildesheim Institut für Mathematik und Angewandte Informatik Samelsonplatz 1, D31141 Hildesheim

E-Mail: girnat@imai.uni-hildesheim.de

Gerda Hagenauer, Prof.in.Dr.in; Professorin für Bildungswissenschaft an der School of Education der Universität Salzburg. Forschungsschwerpunkte: Emotionen, Motivation und soziale Beziehungen in der Schule und Hochschule; Mixed Methods.

University of Salzburg, Erzabt-Klotz-Straße 1, A-5020 Salzburg,

E-Mail: gerda.hagenauer@sbg.ac.at 
Tina Hascher, Prof. Dr., Professorin für Erziehungswissenschaft mit Schwerpunkt Schul- und Unterrichtsforschung an der Universität Bern. Mitglied des Boards des Interfaculty Centre for Educational Research (ICER). Mitwirkung bei der Entwicklung des Kontextfragebogens der Überprüfung des Erreichens der Grundkompetenzen (ÜGK). Forschungsschwerpunkte: Emotionen, Motivation und soziale Prozesse in Schule und Unterricht; Wohlbefinden in der Schule; Gesundheitsförderung; Lehrer*innenbildung.

University of Bern, Fabrikstrasse 8, CH-3012 Bern, Switzerland.

E-Mail: tina.hascher@edu.unibe.ch 